\title{
The number of spanning trees of a graph
}

\author{
Kinkar C Das ${ }^{1}$, Ahmet S Cevik ${ }^{2^{*}}$ and Ismail N Cangul ${ }^{3}$

\section{Dedicated to Professor Hari M Srivastava}

\author{
"Correspondence: \\ sinan.cevik@selcuk.edu.tr \\ ${ }^{2}$ Department of Mathematics, \\ Faculty of Science, Selçuk University, \\ Campus, Konya, 42075, Turkey \\ Full list of author information is \\ available at the end of the article
}

\begin{abstract}
Let $G$ be a simple connected graph of order $n, m$ edges, maximum degree $\Delta_{1}$ and minimum degree $\delta$. Li et al. (Appl. Math. Lett. 23:286-290, 2010) gave an upper bound on number of spanning trees of a graph in terms of $n, m, \Delta_{1}$ and $\delta$ :

$$
t(G) \leq \delta\left(\frac{2 m-\Delta_{1}-\delta-1}{n-3}\right)^{n-3} .
$$

The equality holds if and only if $G \cong K_{1, n-1}, G \cong K_{n}, G \cong K_{1} \vee\left(K_{1} \cup K_{n-2}\right)$ or $G \cong K_{n}-e$, where $e$ is any edge of $K_{n}$. Unfortunately, this upper bound is erroneous. In particular, we show that this upper bound is not true for complete graph $K_{n}$.

In this paper we obtain some upper bounds on the number of spanning trees of graph $G$ in terms of its structural parameters such as the number of vertices $(n)$, the number of edges $(m)$, maximum degree $\left(\Delta_{1}\right)$, second maximum degree $\left(\Delta_{2}\right)$, minimum degree $(\delta)$, independence number $(\alpha)$, clique number $(\omega)$. Moreover, we give the Nordhaus-Gaddum-type result for number of spanning trees.
\end{abstract}

MSC: $05 \mathrm{C} 50 ; 15 \mathrm{~A} 18$

Keywords: graph; spanning trees; independence number; clique number; first Zagreb index

\section{Introduction}

Let $G=(V, E)$ be a simple connected graph with a vertex set $V(G)=\left\{v_{1}, v_{2}, \ldots, v_{n}\right\}$ and an edge set $E(G)$. Its order is $|V(G)|$, denoted by $n$, and its size is $|E(G)|$, denoted by $m$. For $v_{i} \in V(G)$, the degree (= number of the first neighbors) of the vertex $v_{i}$ is denoted by $d_{i}$. The maximum vertex degree is denoted by $\Delta_{1}$, the second maximum by $\Delta_{2}$, and the minimum vertex degree $\delta$. The number of spanning trees of $G$, denoted by $t(G)$, is the total number of distinct spanning subgraphs of $G$ that are trees.

The Laplacian matrix of a graph $G$ is $L(G)=D(G)-A(G)$, where $D(G)$ is the diagonal matrix of vertex degrees, and $A(G)$ is the $(0,1)$-adjacency matrix of graph $G$. Let $\lambda_{1} \geq$ $\lambda_{2} \geq \cdots \geq \lambda_{n}=0$ denote the eigenvalues of $L(G)$. They are usually called the Laplacian eigenvalues of $G$. When more than one graph is under discussion, we may write $\lambda_{i}(G)$ instead of $\lambda_{i}$. For a connected graph of order $n$, it has been proven [1] that

$$
t(G)=\frac{1}{n} \prod_{i=1}^{n-1} \lambda_{i} .
$$

\section{Springer}

(c) 2013 Das et al.; licensee Springer. This is an Open Access article distributed under the terms of the Creative Commons Attribution License (http://creativecommons.org/licenses/by/2.0), which permits unrestricted use, distribution, and reproduction in any medium, provided the original work is properly cited. 
The normalized Laplacian matrix of $G$ is denoted by $\mathcal{L}$ and defined to be

$$
\mathcal{L}=D(G)^{-\frac{1}{2}} L(G) D(G)^{-\frac{1}{2}}
$$

where $L(G)$ is the Laplacian matrix and $D(G)$ is the diagonal matrix of vertex degrees of graph $G$. The eigenvalues of $\mathcal{L}$ are non-negative, we label them so that $0=\rho_{n} \leq \rho_{n-1} \leq$ $\cdots \leq \rho_{2} \leq \rho_{1}$. For a connected graph of order $n$, it has been proven [2] that

$$
t(G)=\frac{1}{2 m} \prod_{i=1}^{n} d_{i} \prod_{i=1}^{n-1} \rho_{i} .
$$

We now give some known popular upper bounds on $t(G)$

1. Grimmett [3].

$$
t(G) \leq \frac{1}{n}\left(\frac{2 m}{n-1}\right)^{n-1}
$$

2. Grone and Merris [4].

$$
t(G) \leq\left(\frac{n}{n-1}\right)^{n-1}\left(\frac{\prod_{i=1}^{n} d_{i}}{2 m}\right)
$$

3. Nosal [5].

$$
t(G) \leq n^{n-2}\left(\frac{r}{n-1}\right)^{n-1}
$$

4. Kelmans [6, p.222].

$$
t(G) \leq n^{n-2}\left(1-\frac{2}{n}\right)^{m}
$$

5. Das [7].

$$
t(G) \leq\left(\frac{2 m-\Delta_{1}-1}{n-2}\right)^{n-2}
$$

The third bound only applies to regular graphs of degree $r$. The first three bounds are sharp for complete graphs only. The fifth bound is sharp for star or complete graph. Moreover, the bound in (5) was also obtained by McKay [8]. Chung et al. [9] studied the number of spanning trees for regular graphs. As usual, $K_{n}, K_{p, q}(p+q=n)$ and $K_{1, n-1}$ denote, respectively, the complete graph, the complete bipartite graph and the star on $n$ vertices.

The paper is organized as follows. In Section 2, we give a list of some previously known results. In Section 3, we obtain some upper bounds on the number of spanning trees. In Section 4, we obtain Nordhaus-Gaddum-type result for the number of spanning trees of graph $G$. 


\section{Lemmas}

In this section, we shall list some previously known results that will be needed in the next two sections. The next lemma is firstly obtained in Theorem 2.6 [7].

Lemma 1 ([7]) Let $G$ be a connected graph of order $n$. Then $\lambda_{1}=\lambda_{2}=\cdots=\lambda_{n-1}$ if and only if $G \cong K_{n}$.

We now give a lower bound on the sum of the largest two Laplacian eigenvalues of graph $G$.

Lemma 2 ([10]) Let $G$ be a connected graph of order $n>2$. Then $\lambda_{1}+\lambda_{2} \geq \Delta_{1}+\Delta_{2}+1$.

Lemma 3 ([10]) Let $G$ be a graph on $n$ vertices, which has at least one edge. Then

$$
\lambda_{1} \geq \Delta_{1}+1
$$

Moreover, if $G$ is connected, then the equality in (8) holds if and only if $\Delta_{1}=n-1$.

A well-known theorem in an algebraic graph theory is the interlacing of the Laplacian spectrum in Theorem 13.6.2 [1].

Lemma 4 ([1]) Let $G$ be a graph of $n$ vertices, and let $H$ be a subgraph of $G$ obtained by deleting an edge in $G$. Then

$$
\lambda_{1}(G) \geq \lambda_{1}(H) \geq \lambda_{2}(G) \geq \lambda_{2}(H) \geq \cdots \geq \lambda_{n-1}(G) \geq \lambda_{n-1}(H) \geq \lambda_{n}(G) \geq \lambda_{n}(H)=0,
$$

where $\lambda_{i}(G)$ is the ith largest Laplacian eigenvalue of $G$, and $\lambda_{i}(H)$ is the ith largest Laplacian eigenvalue of $H$.

Lemma 5 ([11]) Let G be a simple graph with the Laplacian spectrum

$$
\left\{0=\lambda_{n}, \lambda_{n-1}, \ldots, \lambda_{2}, \lambda_{1}\right\}
$$

Then the Laplacian spectrum of $\bar{G}$ is $\left\{0, n-\lambda_{1}, n-\lambda_{2}, \ldots, n-\lambda_{n-2}, n-\lambda_{n-1}\right\}$, where $\bar{G}$ is the complement graph of $G$.

We also have the following result, which is obtained in [12].

Lemma 6 ([12]) Let $G$ be a graph of order $n$ without isolated vertices. Then $\rho_{1}=\rho_{2}=\rho_{3}=$ $\cdots=\rho_{n-1}$ if and only if $G \cong K_{n}$.

The result is the following lemma, known as Kober's inequality.

Lemma 7 ([13]) Let $x_{1}, x_{2}, \ldots, x_{n}$ be non negative numbers, and also let

$$
\alpha=\frac{1}{n} \sum_{i=1}^{n} x_{i} \quad \text { and } \quad \gamma=\left(\prod_{i=1}^{n} x_{i}\right)^{1 / n}
$$


be their arithmetic and geometric means. Then

$$
\frac{1}{n(n-1)} \sum_{i<j}\left(\sqrt{x_{i}}-\sqrt{x_{j}}\right)^{2} \leq \alpha-\gamma \leq \frac{1}{n} \sum_{i<j}\left(\sqrt{x_{i}}-\sqrt{x_{j}}\right)^{2} .
$$

Moreover, the equality holds if and only if $x_{1}=x_{2}=\cdots=x_{n}$.

\section{Bounds on the number of spanning trees}

In [14], an upper bound for $t(G)$ is obtained as follows.

Theorem 1 ([14]) Let $G$ be a connected graph of order $n(n>3)$ with $m$ edges, maximum degree $\Delta_{1}$ and minimum degree $\delta$. Then

$$
t(G) \leq \delta\left(\frac{2 m-\Delta_{1}-\delta-1}{n-3}\right)^{n-3}
$$

The equality holds if and only if $G \cong K_{1, n-1}, G \cong K_{n}, G \cong K_{1} \vee\left(K_{1} \cup K_{n-2}\right)$ or $G \cong K_{n}-e$, where $e$ is any edge of $K_{n}$.

Here we show that Theorem 1 is not true for complete graph $K_{n}$. For this, we need the following lemma.

Lemma 8 For positive integer $a>0$,

$$
\left(1+\frac{1}{a(a+3)}\right)^{a}<1+\frac{1}{a+2}
$$

Proof We have

$$
\begin{aligned}
& \left(1+\frac{1}{a(a+3)}\right)^{a} \\
& \quad=1+\frac{1}{a+3}+\left(\begin{array}{l}
a \\
2
\end{array}\right) \frac{1}{a^{2}(a+3)^{2}}+\left(\begin{array}{l}
a \\
3
\end{array}\right) \frac{1}{a^{3}(a+3)^{3}}+\cdots+\left(\begin{array}{l}
a \\
a
\end{array}\right) \frac{1}{a^{a}(a+3)^{a}} .
\end{aligned}
$$

In fact, this satisfies

$$
\begin{aligned}
& <1+\frac{1}{a+3}+\frac{1}{2 !(a+3)^{2}}+\frac{1}{3 !(a+3)^{3}}+\frac{1}{4 !(a+3)^{4}}+\cdots+\frac{1}{a !(a+3)^{a}} \\
& <1+\frac{1}{a+3}+\frac{1}{a+3}\left(\frac{1}{2(a+3)}+\frac{1}{2^{2}(a+3)^{2}}+\frac{1}{2^{3}(a+3)^{3}}+\cdots+\frac{1}{2^{a-1}(a+3)^{a-1}}\right) \\
& =1+\frac{1}{a+3}+\frac{1}{2(a+3)^{2}} \frac{1-\frac{1}{2^{a-1}(a+3)^{a-1}}}{1-\frac{1}{2(a+3)}} .
\end{aligned}
$$

Now, we have to show that

$$
\frac{1}{a+3}+\frac{1}{2(a+3)^{2}} \cdot \frac{1-\frac{1}{2^{a-1}(a+3)^{a-1}}}{1-\frac{1}{2(a+3)}}<\frac{1}{a+2},
$$


that is,

$$
2^{a-1}(a+3)^{a}>-(a+2)
$$

which is always true, as $a$ is a positive integer. This completes the proof.

Upper bound of $t(G)$ in Theorem 1 is not true for $K_{n}(n>3)$. It is well known that $t\left(K_{n}\right)=$ $n^{n-2}$. Here, we have to show that

$$
(n-1)\left(\frac{n(n-3)+1}{n-3}\right)^{n-3}<t\left(K_{n}\right)=n^{n-2}
$$

Now, putting $a=n-3$ in (9), we get

$$
\left(1+\frac{1}{n(n-3)}\right)^{n-3}<1+\frac{1}{n-1}
$$

which gives result (11).

Hence the correct statement is as follows.

Theorem 2 ([14]) Let $G\left(\neq K_{n}\right)$ be a connected graph of order $n(n>3)$ with m edges, maximum degree $\Delta_{1}$ and minimum degree $\delta$. Then

$$
t(G) \leq \delta\left(\frac{2 m-\Delta_{1}-\delta-1}{n-3}\right)^{n-3}
$$

with the equality holding in (12) if and only if $G \cong K_{1, n-1}, G \cong K_{1} \vee\left(K_{1} \cup K_{n-2}\right)$ or $G \cong K_{n}-e$, where $e$ is any edge of $K_{n}$.

Proof Since $G \nsupseteq K_{n}$, we have $\mu_{n-1} \leq \delta$, where $\delta$ is the minimum degree in $G$. The remaining part of the proof is same as in Theorem 3.1 [14].

We now give an upper bound on the number of spanning trees $t(G)$ in terms of $n, m, \Delta_{1}$ and $\delta$.

Theorem 3 Let $G$ be a connected graph on $n$ vertices with $m$ edges, maximum degree $\Delta_{1}$ and minimum degree $\delta$. Then

$$
t(G) \leq \frac{1}{2 m} \Delta_{1} \delta\left(\frac{2 m-\Delta_{1}-\delta}{n-2}\right)^{n-2}\left(\frac{n}{n-1}\right)^{n-1}
$$

with the equality holding in (13) if and only if $G \cong K_{n}$.

Proof By the arithmetic-geometric mean inequality, we have

$$
\prod_{i=2}^{n-1} d_{i} \leq\left(\frac{2 m-\Delta_{1}-\delta}{n-2}\right)^{n-2} \quad \text { as } 2 m=\sum_{i=1}^{n} d_{i}
$$


and

$$
\prod_{i=2}^{n-1} \rho_{i} \leq\left(\frac{n-\rho_{1}}{n-2}\right)^{n-2} \quad \text { as } n=\sum_{i=1}^{n-1} \rho_{i}
$$

Using the above results in (2), we get

$$
t(G) \leq \frac{1}{2 m} \Delta_{1} \delta\left(\frac{2 m-\Delta_{1}-\delta}{n-2}\right)^{n-2} \rho_{1}\left(\frac{n-\rho_{1}}{n-2}\right)^{n-2}
$$

Let us consider the function

$$
f(x)=x(n-x)^{n-2}, \quad 0 \leq x \leq 2 .
$$

Then we have

$$
f^{\prime}(x)=(n-x)^{n-3}[n-(n-1) x], \quad 0 \leq x \leq 2 .
$$

Thus, $f(x)$ is an increasing function on $\left[0, \frac{n}{n-1}\right]$ and a decreasing function on $\left[\frac{n}{n-1}, 2\right]$. Hence the maximum value of $f(x)$ is

$$
\left(\frac{n}{n-1}\right)^{n-1}(n-2)^{n-2}
$$

Using (14), we get the required result in (13). Thus, the first part of the proof is done.

Now, we suppose that the equality holds in (13). Then all inequalities in the argument above must be equalities. Thus, we have $\rho_{1}=\frac{n}{n-1}$. From the equality in (14), we get $d_{2}=d_{3}=$ $\cdots=d_{n-1}$ and $\rho_{2}=\rho_{3}=\cdots=\rho_{n-1}=\frac{n}{n-1}$. Therefore, $\rho_{1}=\rho_{2}=\rho_{3}=\cdots=\rho_{n-1}$. By Lemma 6, $G \cong K_{n}$.

Conversely, one can easily see that the equality holds in (13) for complete graph $K_{n}$.

Here, we give an upper bound on the number of spanning trees $t(G)$ in terms of $n, m$, $\Delta_{1}$ and $\Delta_{2}$.

Theorem 4 Let $G$ be a connected graph on $n$ vertices, $m$ edges with maximum degree $\Delta_{1}$ and second maximum degree $\Delta_{2}$. Then

$$
t(G)<\frac{1}{4 n(n-3)^{n-3}}\left(\Delta_{1}+\Delta_{2}+1\right)^{2}\left(2 m-\Delta_{1}-\Delta_{2}-1\right)^{n-3}
$$

Proof By the arithmetic-geometric mean inequality, we have

$$
\lambda_{1} \lambda_{2} \leq\left(\frac{\lambda_{1}+\lambda_{2}}{2}\right)^{2}
$$

and

$$
\prod_{i=3}^{n-1} \lambda_{i} \leq\left(\frac{2 m-\lambda_{1}-\lambda_{2}}{n-3}\right)^{n-3} \quad \text { as } 2 m=\sum_{i=1}^{n-1} \lambda_{i}
$$


Using the above results in (1), we get

$$
t(G) \leq \frac{1}{n}\left(\frac{\lambda_{1}+\lambda_{2}}{2}\right)^{2} \cdot\left(\frac{2 m-\lambda_{1}-\lambda_{2}}{n-3}\right)^{n-3}
$$

Let us consider a function

$$
f(x)=x^{2}(2 m-x)^{n-3}
$$

We, thus, have

$$
f^{\prime}(x)=x(2 m-x)^{n-4}(4 m-(n-1) x) .
$$

For $x=\lambda_{1}+\lambda_{2}$, we have $f^{\prime}(x) \leq 0$ as $(n-1) x \geq 4 m=2 \sum_{i=1}^{n-1} \lambda_{i}$. Thus, $f(x)$ is a decreasing function and $\lambda_{1}+\lambda_{2} \geq \Delta_{1}+\Delta_{2}+1$, by Lemma 2 , and hence

$$
t(G) \leq \frac{1}{4 n(n-3)^{n-3}}\left(\Delta_{1}+\Delta_{2}+1\right)^{2}\left(2 m-\Delta_{1}-\Delta_{2}-1\right)^{n-3}
$$

By contradiction, we will show that the inequality in (17) is strict. Suppose that the equality holds in (17). Then all the inequalities in the argument above must be equalities. Thus, we have $\lambda_{1}+\lambda_{2}=\Delta_{1}+\Delta_{2}+1$. From equality in (16), we get $\lambda_{1}=\lambda_{2}$ and $\lambda_{3}=\lambda_{4}=\cdots=\lambda_{n-1}$. By Lemma 3, we have $\Delta_{1}+\Delta_{2}+1=\lambda_{1}+\lambda_{2}=2 \lambda_{1} \geq 2\left(\Delta_{1}+1\right) \geq \Delta_{1}+\Delta_{2}+2$, a contradiction.

This completes the proof.

For $1 \leq \alpha \leq n-1$, let $C I(n, \alpha)$ be a split graph on $n$ vertices consisting of a $\bar{K}_{\alpha}$ (complement of the complete graph on $\alpha$ vertices) and a $K_{n-\alpha}$ (complete graph on the remaining $n-\alpha$ vertices), in which each vertex of the $\bar{K}_{\alpha}$ is adjacent to each vertex of the $K_{n-\alpha}$. Therefore,

$$
C I(n, \alpha)=K_{n-\alpha} \vee \bar{K}_{\alpha}
$$

We now give another upper bound on the number of spanning trees in terms of $n$ and $\alpha$.

Theorem 5 Let $G$ be a simple connected graph of order $n$ with an independence number $\alpha$. Then

$$
t(G) \leq n^{n-\alpha-1}(n-\alpha)^{\alpha-1}
$$

with the equality holding in (18) if and only if $G \cong C I(n, \alpha)$.

Proof By Lemma 4, we have

$$
\lambda_{i}(G+e) \geq \lambda_{i}(G), \quad i=1,2, \ldots, n,
$$

where $e$ is an edge. So if we add one by one edges in $G$ such that independence number $\alpha$ is fixed of the resultant graph, then finally, we obtain a split graph $C I(n, \alpha)$. One can easily 
see that

$$
t(G) \leq t(C I(n, \alpha))=n^{n-\alpha-1}(n-\alpha)^{\alpha-1}
$$

as Laplacian spectrum of $\overline{C I}(n, \alpha)$ is $\underbrace{\alpha, \alpha, \ldots, \alpha}_{\alpha-1}, \underbrace{0,0, \ldots, 0}_{n-\alpha+1}$, that is, Laplacian spectrum of $C I(n, \alpha)$ is $\underbrace{n, n, \ldots, n}_{n-\alpha}, \underbrace{n-\alpha, n-\alpha, \ldots, n-\alpha}_{\alpha-1}, 0$, by Lemma 5 .

Since $G$ is connected, one can easily see that

$$
t(G+e)>t(G)
$$

This completes the proof of this theorem.

We now give another upper bound on $t(G)$ in terms of $n, m$ and $\omega$.

Theorem 6 Let $G$ be a connected graph of order $n, m$ edges and clique number $\omega$. Then

$$
t(G) \leq \frac{\omega^{\omega-2}(2 m-\omega(\omega-2))^{n-\omega+1}}{n(n-\omega+1)^{n-\omega+1}}
$$

with the equality holding if and only if $G \cong K_{n}$.

Proof By the arithmetic-geometric mean inequality, we have

$$
\prod_{i=1}^{\omega-2} \lambda_{i} \leq\left(\frac{\sum_{i=1}^{\omega-2} \lambda_{i}}{\omega-2}\right)^{\omega-2} \text { and } \prod_{i=\omega-1}^{n-1} \lambda_{i} \leq\left(\frac{\sum_{i=\omega-1}^{n-1} \lambda_{i}}{n-\omega+1}\right)^{n-\omega+1}
$$

Since $\omega$ is the clique number of $G$, by using (1), we get

$$
\begin{aligned}
t(G)= & \frac{1}{n} \prod_{i=1}^{\omega-2} \lambda_{i} \prod_{i=\omega-1}^{n-1} \lambda_{i} \leq \frac{1}{n}\left(\frac{\sum_{i=1}^{\omega-2} \lambda_{i}}{\omega-2}\right)^{\omega-2} \times\left(\frac{\sum_{i=\omega-1}^{n-1} \lambda_{i}}{n-\omega+1}\right)^{n-\omega+1} \\
= & \frac{1}{n(\omega-2)^{\omega-2}(n-\omega+1)^{n-\omega+1}} A^{\omega-2}(2 m-A)^{n-\omega+1} \\
& \text { where } A=\sum_{i=1}^{\omega-2} \lambda_{i} .
\end{aligned}
$$

Let us consider a function

$$
f(x)=x^{\omega-2}(2 m-x)^{n-\omega+1} .
$$

Then, we have

$$
f^{\prime}(x)=x^{\omega-3}(2 m-x)^{n-\omega}(2 m(\omega-2)-(n-1) x) .
$$

Since $\lambda_{1} \geq \lambda_{2} \geq \cdots \geq \lambda_{n-1}$, we have

$$
(n-\omega+1) \sum_{i=1}^{\omega-2} \lambda_{i} \geq(n-\omega+1)(\omega-2) \lambda_{\omega-2} \geq(\omega-2) \sum_{i=\omega-1}^{n-1} \lambda_{i},
$$


that is,

$$
(n-1) A=(n-1) \sum_{i=1}^{\omega-2} \lambda_{i} \geq(\omega-2) \sum_{i=1}^{n-1} \lambda_{i}=2 m(\omega-2) .
$$

By using this inequality above, we conclude that $f(x)$ is a decreasing function, as $f^{\prime}(x) \leq 0$. Since $\omega$ is a clique number of $G$, we must have $\lambda_{i} \geq \omega, i=1,2, \ldots, \omega-1$, and hence $A=\sum_{i=1}^{\omega-2} \lambda_{i} \geq \omega(\omega-2)$. Thus, we have

$$
f(x) \leq \omega^{\omega-2}(\omega-2)^{\omega-2}(2 m-\omega(\omega-2))^{n-\omega+1} .
$$

Using the above result with (20), we get the required result (19). The first part of the proof is done.

Now, we suppose that the equality holds in (19). Then all the inequalities in the argument above must be equalities. Thus, we have $\lambda_{1}=\lambda_{2}=\cdots=\lambda_{\omega-2}=\omega$ and $\lambda_{\omega-1}=\lambda_{\omega}=\cdots=$ $\lambda_{n-1}=\omega$. Hence $\lambda_{i}=\omega, i=1,2, \ldots, n-1$. By Lemma $1, G \cong K_{n}$.

Conversely, one can easily see that the equality holds in (19) for complete graph $K_{n}$.

The first Zagreb index $M_{1}(G)$ is defined as follows:

$$
M_{1}(G)=\sum_{i=1}^{n} d_{i}^{2}
$$

The first Zagreb index $M_{1}(G)$ was introduced in [15] and elaborated in [16]. The main properties of $M_{1}(G)$ were summarized in [17]. Some recent results on the first Zagreb index are reported in [18-21]. Now, we are ready to give some lower and upper bounds on the number of spanning trees.

Theorem 7 Let $G$ be a connected graph of order $n$ with $m$ edges and first Zagreb index $M_{1}(G)$. Then

$$
t(G) \geq \frac{1}{n}\left[\frac{4 m^{2}-(n-2)\left(M_{1}(G)+2 m\right)}{n-1}\right]^{\frac{n-1}{2}}
$$

with the equality holding in (21) if and only if $G \cong K_{n}$. Moreover,

$$
t(G) \leq \frac{1}{n}\left[\frac{4 m^{2}-M_{1}(G)+2 m}{(n-1)(n-2)}\right]^{\frac{n-1}{2}}
$$

with the equality holding in (22) if and only if $G \cong K_{n}$.

Proof We have

$$
\begin{aligned}
\sum_{i<j}\left(\lambda_{i}-\lambda_{j}\right)^{2} & =\frac{1}{2} \sum_{i=1}^{n-1} \sum_{j=1}^{n-1}\left(\lambda_{i}^{2}+\lambda_{j}^{2}-2 \lambda_{i} \lambda_{j}\right) \\
& =\frac{1}{2}\left[(n-1) \sum_{i=1}^{n-1} \lambda_{i}^{2}+(n-1) \sum_{j=1}^{n-1} \lambda_{j}^{2}-2 \sum_{i=1}^{n-1} \lambda_{i} \sum_{j=1}^{n-1} \lambda_{j}\right]
\end{aligned}
$$




$$
\begin{aligned}
& =(n-1) \sum_{i=1}^{n-1} \lambda_{i}^{2}-\left(\sum_{i=1}^{n-1} \lambda_{i}\right)^{2}=(n-1) \sum_{i=1}^{n} d_{i}\left(d_{i}+1\right)-\left(\sum_{i=1}^{n} d_{i}\right)^{2} \\
& \quad \text { as } \sum_{i=1}^{n-1} \lambda_{i}^{2}=\sum_{i=1}^{n} d_{i}\left(d_{i}+1\right) \text { and } \sum_{i=1}^{n-1} \lambda_{i}=\sum_{i=1}^{n} d_{i} \\
& =(n-1)\left(M_{1}(G)+2 m\right)-4 m^{2} \quad \text { as } \sum_{i=1}^{n} d_{i}=2 m .
\end{aligned}
$$

Since $G$ is connected, $\lambda_{n-1}>0$. Now, by setting $x_{i}=\lambda_{i}^{2}, i=1,2, \ldots, n-1$ and by Lemma 7 , we obtain

$$
\frac{\sum_{i=1}^{n-1} \lambda_{i}^{2}}{n-1}-\left(\prod_{i=1}^{n-1} \lambda_{i}^{2}\right)^{1 / n-1} \leq M_{1}(G)+2 m-\frac{4 m^{2}}{n-1}, \quad \text { by }(23)
$$

that is, by considering (1),

$$
\frac{\sum_{i=1}^{n} d_{i}\left(d_{i}+1\right)}{n-1}-(n t(G))^{2 / n-1} \leq M_{1}(G)+2 m-\frac{4 m^{2}}{n-1}
$$

since $\sum_{i=1}^{n-1} \lambda_{i}^{2}=\sum_{i=1}^{n} d_{i}\left(d_{i}+1\right)$. From this last inequality, we then get

$$
(n t(G))^{2 / n-1} \geq \frac{4 m^{2}}{n-1}-\left(\frac{n-2}{n-1}\right)\left(M_{1}(G)+2 m\right), \quad \text { as } M_{1}(G)=\sum_{i=1}^{n} d_{i}^{2}
$$

which gives the required result (21). Similarly, by Lemma 7, we obtain

$$
(n t(G))^{2 / n-1} \leq \frac{1}{(n-1)(n-2)}\left(4 m^{2}-M_{1}(G)+2 m\right),
$$

as required in (22). Hence the first part of the proof is completed.

Now, we suppose that the equality holds in (21) or (22). Then all the inequalities in the argument above must be equalities. By Lemma 7, we have $\lambda_{1}=\lambda_{2}=\lambda_{3}=\cdots=\lambda_{n-1}$. By Lemma 1 , we get $G \cong K_{n}$.

Conversely, one can easily see that the equalities in (21) and (22) hold for complete graphs $K_{n}$.

Example 1 For the three graphs $G_{1}, G_{2}$ and $G_{3}$ in Figure $1, t\left(G_{1}\right), t\left(G_{2}\right)$ and $t\left(G_{3}\right)$ are 3, 8 and 9 , respectively. The numerical results related to the bounds (that were mentioned above) are listed in the following. At this point, we should note that these results are presenting as rounded the one decimal place.

$\begin{array}{ccccccccccc} & t(G) & (3) & (4) & (7) & (12) & (13) & (15) & (18) & (19) & (22) \\ G_{1} & 3 & 7.8 & 3.9 & 4.6 & 4 & 4.5 & 5.5 & 20 & 7.6 & 9.8 \\ G_{2} & 8 & 16.2 & 9.8 & 12.7 & 9 & 10.3 & 12.8 & 20 & 16.2 & 20.7 \\ G_{3} & 9 & 16.2 & 13 & 12.7 & 12.5 & 13 & 20 & 75 & 16.2 & 21.4\end{array}$




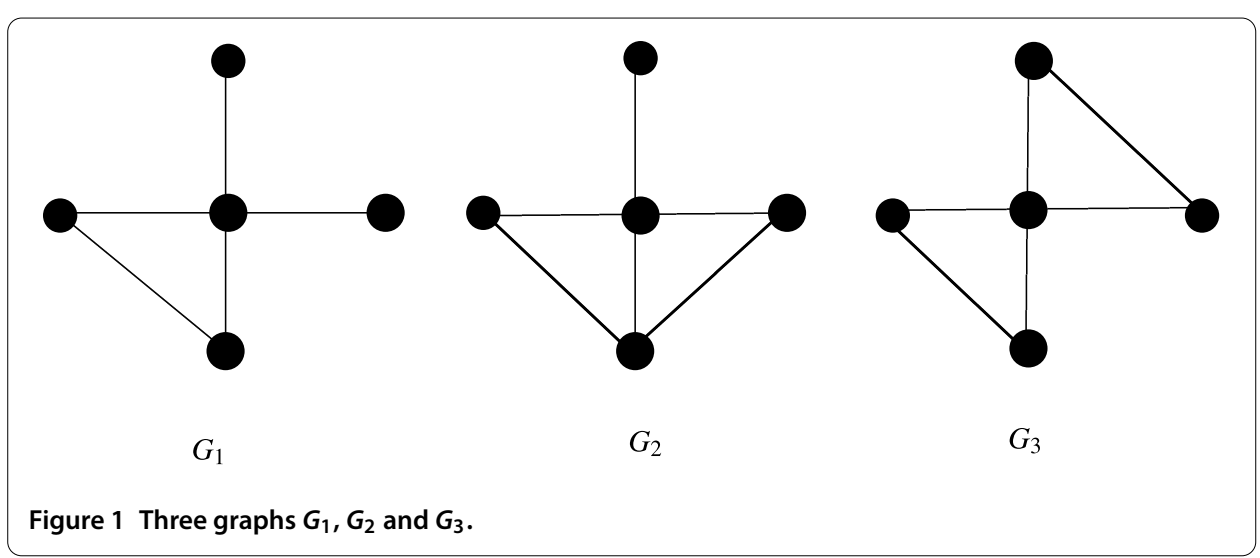

\section{Nordhaus-Gaddum-type results for the number of spanning trees of a graph}

For a graph $G$, the chromatic number $\chi(G)$ is the minimum number of colors needed to color the vertices of $G$ in such a way that no two adjacent vertices are assigned the same color. In 1956, Nordhaus and Gaddum [22] gave bounds involving the chromatic number $\chi(G)$ of a graph $G$ and its complement $\bar{G}$ :

$$
2 \sqrt{n} \leq \chi(G)+\chi(\bar{G}) \leq n+1 .
$$

Motivated by the results above, we now obtain analogous conclusions for the number of spanning trees.

Theorem 8 Let $G$ be a connected graph on $n \geq 4$ vertices and $m$ edges with a connected complement $\bar{G}$. Then

$$
\begin{aligned}
t(G) & +t(\bar{G}) \\
\leq & \frac{1}{n(n-2)^{n-2}} \\
& \times\left[\left(\Delta_{1}+1\right)\left(2 m-\Delta_{1}-1\right)^{n-2}+\left(n-\Delta_{1}-1\right)\left(n(n-2)-2 m+\Delta_{1}+1\right)^{n-2}\right],
\end{aligned}
$$

where $\Delta_{1}$ is the maximum degree in $G$.

Proof By Lemma 5, from (1), we have

$$
\begin{aligned}
t(G)+t(\bar{G}) & =\frac{1}{n} \prod_{i=1}^{n-1} \lambda_{i}+\frac{1}{n} \prod_{i=1}^{n-1}\left(n-\lambda_{i}\right) \\
& \leq \frac{1}{n}\left[\lambda_{1}\left(\frac{2 m-\lambda_{1}}{n-2}\right)^{n-2}+\left(n-\lambda_{1}\right)\left(\frac{n(n-2)-2 m+\lambda_{1}}{n-2}\right)^{n-2}\right]
\end{aligned}
$$

by the arithmetic-geometric mean inequality

$$
=\frac{1}{n(n-2)^{n-2}}\left[\lambda_{1}\left(2 m-\lambda_{1}\right)^{n-2}+\left(n-\lambda_{1}\right)\left(n(n-2)-2 m+\lambda_{1}\right)^{n-2}\right] .
$$


Let us consider a function

$$
f(x)=x(2 m-x)^{n-2}+(n-x)(n(n-2)-2 m+x)^{n-2} \text { for } \Delta_{1}+1 \leq x \leq n .
$$

We have

$$
\begin{aligned}
f^{\prime}(x) & =(2 m-x)^{n-3}(2 m-(n-1) x)-(n(n-2)-2 m+x)^{n-3}((n-1) x-2 m) \\
& =-((n-1) x-2 m)\left[(2 m-x)^{n-3}+(n(n-2)-2 m+x)^{n-3}\right]<0 .
\end{aligned}
$$

Thus, $f(x)$ is a decreasing function on $\Delta_{1}+1 \leq x \leq n$. Using the result above in (25), we obtain the required result (24).

The next result presents another upper bound for $t(G)+t(\bar{G})$. In fact, the proof of it is clear by considering Theorem 7 .

Theorem 9 Let $G$ be a graph on $n$ vertices and $m$ edges. Then

$$
\begin{aligned}
t(G) & +t(\bar{G}) \\
\leq & \frac{1}{n(n-1)^{(n-1) / 2}(n-2)^{(n-1) / 2}}\left[\left(4 m^{2}-M_{1}(G)+2 m\right)^{(n-1) / 2}\right. \\
& \left.+\left(n(n-1)\left(n^{2}-2 n+2\right)+2 m\left(2 m-2(n-1)^{2}-1\right)-M_{1}(G)\right)^{(n-1) / 2}\right],
\end{aligned}
$$

where $M_{1}(G)$ is the first Zagreb index of graph G. Moreover, the equality in (26) holds if and only if $G \cong K_{n}$ or $\bar{G} \cong K_{n}$.

\section{Competing interests}

The authors declare that they have no competing interests.

\section{Authors' contributions}

All authors completed the paper together. All authors read and approved the final manuscript.

\section{Author details}

${ }^{1}$ Department of Mathematics, Sungkyunkwan University, Suwon, 440-746, Republic of Korea. ${ }^{2}$ Department of Mathematics, Faculty of Science, Selçuk University, Campus, Konya, 42075, Turkey. ${ }^{3}$ Department of Mathematics, Faculty of Arts and Science, Uludag University, Gorukle Campus, Bursa, 16059, Turkey.

\section{Acknowledgements}

The authors are grateful to the referees for their valuable comments, which lead to an improvement of the original manuscript. This paper was prepared during the first author's visit in Selcuk and Uludag Universities. Moreover, we are thankful to Mr. SA Mojallal for computing the values in Example 1. The first author is supported by the Faculty research Fund, Sungkyunkwan University, 2012 and the National Research Foundation funded by the Korean government with the grant no. 2013R1A1A2009341. The second and the third authors are both partially supported by the Research Project Offices of Selcuk and Uludag Universities, and TUBITAK (The Scientific and Technological Research Council of Turkey).

Received: 19 November 2012 Accepted: 31 July 2013 Published: 20 August 2013

\footnotetext{
References

1. Godsil, C, Royle, G: Algebraic Graph Theory. Graduate Texts in Mathematics, vol. 207. Springer, Berlin (2001)

2. Chung, FRK: Spectral Graph Theory. Am. Math. Soc., Providence (1997)

3. Grimmett, GR: An upper bound for the number of spanning trees of a graph. Discrete Math. 16, 323-324 (1976)

4. Grone, R, Merris, R: A bound for the complexity of a simple graph. Discrete Math. 69, 97-99 (1988)

5. Nosal, E: Eigenvalues of Graphs. Master thesis, University of Calgary (1970)

6. Cvetković, DM, Doob, M, Sachs, H: Spectra of Graphs. Pure and Applied Mathematics, vol. 87. Academic press, San Diego (1980)

7. Das, KC: A sharp upper bound for the number of spanning trees of a graph. Graphs Comb. 23, 625-632 (2007)
} 
8. McKay, BD: Spanning trees in regular graphs. Eur. J. Comb. 4, 149-160 (1983)

9. Chung, F, Yau, S-T: Coverings, heat kernels and spanning trees. Electron. J. Comb. 6, R12 (1999)

10. Grone, R, Merris, R: The Laplacian spectrum of a graph II. SIAM J. Discrete Math. 7(2), 221-229 (1994)

11. Merris, R: Laplacian matrices of graphs: a survey. Linear Algebra Appl. 197(198), 143-176 (1994)

12. Das, KC, Maden, AD, Cevik, AS: Sharp upper bounds on the spectral radius of the Signless Laplacian matrix of a graph. Appl. Math. Comput. 219(10), 5025-5032 (2013)

13. Kober, H: On the arithmetic and geometric means and on Holder's inequality. Proc. Am. Math. Soc. 59, 452-459 (1958)

14. Li, J, Shiu, WC, Chang, A: The number of spanning trees of a graph. Appl. Math. Lett. 23, 286-290 (2010)

15. Gutman, I, Trinajstić, N: Graph theory and molecular orbitals. Total $\pi$-electron energy of alternant hydrocarbons. Chem. Phys. Lett. 17, 535-538 (1972)

16. Gutman, I, Ruščić, B, Trinajstić, N, Wilcox, CF: Graph theory and molecular orbitals. XII. Acyclic polyenes. J. Chem. Phys. 62, 3399-3405 (1975)

17. Nikolić, S, Kovačević, G, Milićević, A, Trinajstić, N: The Zagreb indices 30 years after. Croat. Chem. Acta 76, 113-124 (2003)

18. Das, KC: Maximizing the sum of the squares of the degrees of a graph. Discrete Math. 285, 57-66 (2004)

19. Das, KC, Gutman, I: Some properties of the second Zagreb index. MATCH Commun. Math. Comput. Chem. 52, 103-112(2004)

20. Das, KC: On comparing Zagreb indices of graphs. MATCH Commun. Math. Comput. Chem. 63(2), $433-440$ (2010)

21. Das, KC, Gutman, I, Zhou, B: New upper bounds on Zagreb indices. J. Math. Chem. 46(2), 514-521 (2009)

22. Nordhaus, EA, Gaddum, JW: On complementary graphs. Am. Math. Mon. 63, 175-177 (1956)

doi:10.1186/1029-242X-2013-395

Cite this article as: Das et al.: The number of spanning trees of a graph. Journal of Inequalities and Applications 2013 2013:395.

\section{Submit your manuscript to a SpringerOpen ${ }^{\ominus}$ journal and benefit from:}

- Convenient online submission

- Rigorous peer review

- Immediate publication on acceptance

- Open access: articles freely available online

- High visibility within the field

- Retaining the copyright to your article 